\title{
Modeling the spectral energy distribution of galaxies
}

\section{Disk opacity and star formation in 5 edge-on spirals}

\author{
A. Misiriotis ${ }^{1,2}$, C. C. Popescu ${ }^{3,4,5, \star}$, R. Tuffs ${ }^{3}$, and N. D. Kylafis ${ }^{1,6}$ \\ 1 University of Crete, Physics Department, PO Box 2208, 71003 Heraklion, Crete, Greece \\ 2 Observatoire de Marseille, 2 place Le Verrier 13248 Marseille Cedex 4, France \\ 3 Max Planck Institut für Kernphysik, Saupfercheckweg 1, 69117 Heidelberg, Germany \\ 4 The Observatories of the Carnegie Institution of Washington, 813 Santa Barbara Street, Pasadena, \\ California 91101, USA \\ 5 Max Planck Institut für Astronomie, Königstuhl 17, 69117 Heidelberg, Germany \\ ${ }^{6}$ Foundation for Research and Technology-Hellas, PO Box 1527, 71110 Heraklion, Crete, Greece
}

Received 27 March 2001 / Accepted 19 April 2001

\begin{abstract}
Using tools previously described and applied to the prototype galaxy NGC 891, we model the optical to far-infrared spectral energy distributions (SED) of four additional edge-on spiral galaxies, namely NGC 5907, NGC 4013, UGC 1082 and UGC 2048. Comparing the model predictions with IRAS and, where available, submillimeter and millimeter observations, we determine the respective roles of the old and young stellar populations in grain heating. In all cases, the young population dominates, with the contribution of the old stellar population being at most $40 \%$, as previously found for NGC 891 . After normalization to the disk area, the massive starformation rate (SFR) derived using our SED modeling technique, which is primarily sensitive to the non-ionizing ultraviolet output from the young stellar population, lies in the range $7 \times 10^{-4}-2 \times 10^{-2} M_{\odot} \mathrm{yr}^{-1} \mathrm{kpc}^{-2}$. This is consistent with normalized SFRs derived for face-on galaxies of comparable surface gas densities from $\mathrm{H}_{\alpha}$ observations. Though the most active star-forming galaxy of the five in absolute terms, NGC 891 is not an exceptional system in terms of its surface density in SFR.
\end{abstract}

Key words. dust, extinction - galaxies: spiral - galaxies: stellar content - galaxies: ISM - infrared: galaxies submillimeter

\section{Introduction}

Optical and Far-Infrared (FIR)/sub-millimeter (submm) data from galaxies contain complementary information about the distribution of stars and dust, from which intrinsic quantities of interest - the star-formation rate (SFR) and star-formation history - can in principle be extracted. This is especially relevant to systems having intermediate optical depths to starlight, or to inhomogeneous systems with optically thin and thick components. Many, perhaps most, of normal (non-starburst) gas-rich galaxies in the local universe may fall into these categories.

On the one hand, optical data probes the colour and spatial distribution (after correction for extinction) of the

Send offprint requests to: A. Misiriotis,

e-mail: angmis@physics.uoc.gr

* Research Associate at The Astronomical Institute of the Romanian Academy, Str. Cuţitul de Argint 5, 75212 Bucharest, Romania. photospheric emission along sufficiently transparent lines of sight. This is particularly useful to investigate older, redder stellar populations in galaxian disks with scale heights larger than that of the dust. A radiation transfer modeling technique that can be applied to edge-on systems, where the scale height of the stars and dust extinction can be directly constrained, was introduced by Kylafis \& Bahcall (1987) and subsequently applied on several galaxies (Xilouris et al. 1997, 1998, 1999, hereafter referred to as X97, X98, X99 respectively). Radiative transfer codes in combination with observations of nearby edge-on galaxies were also used by Ohta \& Kodaira (1995) and Kuchinski et al. (1998).

On the other hand, grains act as test particles probing the strength and colour of ultraviolet (UV)/optical interstellar radiation fields. This constitutes an entirely complementary constraint to studies of photospheric emission. In the FIR, grains are moreover detectable over the full range of optical depths present in a galaxy. At least part of this regime is inaccessible to direct probes of starlight, 
especially at shorter wavelengths, even for face-on systems. This particularly applies to light from young stars located in, or close by, the dust clouds from which they formed, since a certain fraction of the light is locally absorbed. Furthermore, there is at least a possibility that most of the remaining UV and even blue light from young stars that can escape into the disk might be absorbed by diffuse dust there. Observations from IRAS, IRAM (e.g. Neininger et al. 1996; Dumke et al. 1997), SCUBA (e.g. Alton et al. 1998; Israel et al. 1999; Dunne 2000; Bianchi et al. 2000a) and ISO (e.g. Haas et al. 1998; Stickel et al. 2000) can provide information on the quantity and spatial distribution of the dust within spiral galaxies.

A combined analysis of the whole UV-optical/FIR/ submm/mm spectral energy distribution (SED) of galaxies seems to be a promising way to constrain the problem. Radiative transfer codes for an assumed "sandwich" configuration of dust and stars were applied by $\mathrm{Xu} \&$ Buat (1995) and Xu \& Helou (1996) to account for the energy budget over the whole spectral range. They considered in detail the relative contribution of the non-ionizing UV photons and the optical photons in heating the grains. However these calculations did not incorporate a model for the dust grain emission, nor for radial variations in the absorbed radiation in the disk, and therefore could not account for the exact shape of the FIR SED. Recently, there have been several works modeling the SED of galaxies from UV to submm (Silva et al. 1998; Devriendt et al. 1999), by applying photometric and/or spectrophotometric and chemical evolution models of galaxies. While these models are adequate in describing the general shape of the volume-integrated SED, they make use of many free parameters and of a simplified geometry.

Bianchi et al. (2000b) attempted to model NGC 6946 from the UV to FIR using a 3D Monte Carlo radiativetransfer code for a simplified geometry of emitters (a single stellar disk). They concluded that the total FIR output is consistent with an optically thick solution. However, their model did not consider a grain size distribution for grains, stochastic emission of small grains, and the contribution of localized sources within star-forming complexes. This resulted in a poor fit of the FIR SED and a failure to reproduce the IRAS flux densities.

Another work on modeling the UV to submm emission was presented by Efstathiou et al. (2000) for star burst galaxies which were treated as an ensemble of optically thick giant molecular clouds centrally illuminated by recently formed stars. This modeling technique successfully reproduced the observed SED of M 82 and NGC 6090. Such a technique obviously cannot be applied to normal "quiescent" disk galaxies dominated by emission from the diffuse interstellar radiation field.

In Popescu et al. (2000, hereafter referred to as Paper I) we gave a detailed description of a new tool for a combined analysis of the optical-FIR/submm SED of galaxies. This tool included solving the radiativetransfer problem for a realistic distribution of absorbers and emitters and by considering realistic models for dust, taking into account the grain size distribution and stochastic heating of small grains as well as the contribution of HII regions. We applied this tool to the edge-on system NGC 891.

In short, we used the intrinsic spatial distribution of dust and stars (in the $B, V, I, J, K$ bands) derived for this galaxy by X99 as a constraint on the old stellar population and part of the dust distribution. In order to fully explain the optical-FIR-submm SED, it was found necessary to add to the components of the X99's solution both a young stellar population (to correct for a shortfall in FIR brightness in terms of re-radiated non-ionizing UV) and more dust mass (to correct for a shortfall in submm brightness). Our solution explained both the observed SED in the FIR and submm, as well as the observed radial profile at $850 \mu \mathrm{m}$. We found that the dust is predominanly heated by the young stellar population.

Although NGC 891 was chosen for study in Paper I as the prototypical and most extensively observed edge-on galaxy, it exhibits some extreme features, raising the possibility that it might not in fact be representative of a "typical" spiral. In this paper we extend our SED modeling technique to four additional edge-on systems - NGC 5907, NGC 4013, UGC 1082 and UGC 2048 - with the aim of examining whether the features of the solution we obtained for NGC 891 might be more generally applicable. Radiation transfer solutions accounting for the appearance of these four galaxies in the optical bands have been obtained by X99, X98 and X97. We use these solutions to constrain our model for the optical-FIR SEDs in the same fashion as for NGC 891.

The paper is arranged as follows: in Sect. 2 we overview the model and its application to the objects in the present work. In Sect. 3 we present predictions for the FIR SED of all five galaxies studied. These are obtained using our "standard model", i.e. a model with radiation fields derived from the radiation transfer solution for the optical appearance supplemented by a hidden population of young stars embedded in HII regions, but with no additional dust. In Sect. 4 we describe and discuss the SED of one of our four galaxies, namely NGC 5907, in terms of the more complex "two-dust-disk model" and compare this with the corresponding solution obtained for NGC 891 in Paper I. In Sect. 5 we discuss the dependence of the derived star-formation rates on the assumptions of the model, in particular the assumed geometries for the dust and stars. We show that after normalization to disk area, NGC 891 has comparable star-formation characteristics to the other four objects. We also compare the disk-area normalized massive star-formation rate in the five edgeon galaxies, obtained from the optical-FIR SED analysis, with the same quantity derived from $\mathrm{H}_{\alpha}$ measurements of statistical samples of face-on galaxies given in the literature. In Sect. 6 we give a summary of our work.

\section{Model calculations for the five galaxies}

A full description of our model and its application to NGC 891 can be found in Paper I. Here we give an 
overview of the basic idea and its specific application to the four galaxies.

To determine the propagation of light in a galaxy we take both emitters and absorbers to be smoothly arranged as a superposition of simple cylindrically symmetric geometrical distributions. The assumption of an axisymmetric model and the neglect of the spiral structure does not seem to introduce a systematic error on the estimate of the overall opacity, at least when a statistical sample of edge-on galaxies is considered (Misiriotis et al. 2000). The emitters are divided into a young (predominantly UVemitting) stellar population, distributed in a disk with a small scale height, an old (predominantly optical-NIR emitting) population, distributed in a disk with a larger scale height and a bulge. The absorbers are arranged in "young" and "old dust disks", associated with the old and young stellar populations. The "old dust disk" has a larger scale height than the "young dust disk", though not so large as the old stellar disk. Both the stellar- and dust-disk distributions are specified as exponential density distributions in radius and height, while the stellar bulge is described by a de Vaucouleurs law. All radial distributions have a common truncation at three scale lengths of the "old dust disk".

The first step in the calculation of the optical-FIR SED is to determine the geometric and amplitude parameters for the old stellar disk and bulge independently in each band pass ranging from the $B$ band to the NIR for a common "old dust disk" (whose geometry and opacity is also determined). This can be achieved by a radiation transfer analysis matching as closely as possible the predicted and observed brightness distributions, following Kylafis \& Bahcall (1987) and including inclination as a free parameter. For the five galaxies discussed in this work we adopt the solutions given in X97 and X99 on the basis of observations in $B, V$ and $I$, and in the case of NGC 891 also in $J$ and $K$ bands.

This analysis of the optical-NIR emission also yields the extinction coefficient as a function of wavelength for the "old dust disk". For all five galaxies studied, the measured extinction law was found to be close to the predicted law derived from the dust model used in the calculation of the IR emission. This corresponds to the graphite-silicate mix and dust size distribution determined for the Galaxy by Mathis et al. (1977) (see Eqs. (2) and (3) of Paper I) with optical constants taken from Laor \& Draine (1993). When calculating the IR emission we take grains in the "young dust disk", if present, to have the same size distribution and optical properties as those in the "old dust disk".

To this fixed basis for the old stellar population and associated dust disk, the model allows the addition of a young, UV-emitting stellar population and, optionally, associated dust. The additional stars and dust are taken to be distributed in a common exponential disk with a scalelength equal to that of the already determined intrinsic $B$-band population, and a scale height fixed at $90 \mathrm{pc}$ (as for the Milky Way). Since this scale height is typically a factor of several times smaller than the scale heights derived by X97 and X99 for the "old dust disk", this constitutes the simplest assumption for the distribution of these extra components, which hide UV-optical indicators of the young stellar population and associated additional opacity. As emphasized in Paper I, a clumpy distribution for the dust associated with the young stellar population is also possible, though very difficult to calculate, and, due to the lack of high angular resolution FIR images of spiral galaxies, at present not directly verifiable. The luminosity of the young stars is a free parameter of the model, which we express as a recent star-formation rate SFR, based on the population synthesis models of Bruzual \& Charlot (2001) for $Z=Z_{\odot}$, a Salpeter IMF with a cut-off mass of $100 M_{\odot}$ and $S F R=(1 / \tau) \exp (-t / \tau)$ with $\tau=5$ Gyr.

The model also incorporates a parameter $F$ representing the fraction of the non-ionizing UV luminosity from the young stellar population, which is locally absorbed in star-formation regions by optically thick fragments of their parent molecular clouds. This fraction of the luminosity is considered to be re-radiated in the FIR according to a spectral template matching the observed spectrum of galactic HII regions. This template generally has a warmer $60 / 100 \mu \mathrm{m}$ colour than that calculated for the diffuse disk.

Given the intrinsic distributions of stellar emissivities in the UV, optical and NIR, and the distribution of absorbers in the "old" and (if present) the "young dust disks", we then proceed with a radiative transfer calculation to determine the UV-NIR energy density of the radiation field throughout the galaxy. In the absence of solutions in the $J$ and $K$ bands, we extrapolate the energy density from the $B, V, I$ bands assuming a black-body spectral distribution and a colour temperature of $4000 \mathrm{~K}$. In calculating the heating of grains placed in the resulting radiation field, we include an explicit treatment of stochastic emission as detailed in Paper I. The IR-submm emission from grains is then calculated for a grid of positions in the galaxy. Subsequently we integrate over the entire galaxy to obtain the IR-submm SED of the diffuse disk emission. Prior to comparison with observed FIR-submm SEDs, the spectral template for the HII regions, scaled according to the value of $F$, must be added to this calculated spectral distribution of diffuse FIR emission.

Due to the precise constraints on the distribution of stellar emissivity in the optical-near infrared (NIR) and the distribution of dust in the "old dust disk" yielded by the radiation transfer analysis of the highly resolved optical-NIR images, coupled with the simple assumptions for the distribution of the young stellar population and associated dust, our model has a maximum of three free parameters from which the FIR-submm SED can be fully determined. These are the SFR for massive stars, the parameter $F$ representing the fraction of the non-ionizing UV luminosity from massive stars which is locally absorbed, and the mass of dust in the "young dust disk". In general terms, the last parameter is principally constrained by the submm emission, the non-ionizing UV luminosity by the bolometric FIR-submm output and the 
Table 1. Properties of the galaxies used in the modeling of the FIR-submm SEDs. The dust mass is that quoted in X97 and X99 (corresponding to the "old dust disk" in this paper). The submm fluxes are taken from ${ }^{1}$ Alton et al. (1998), ${ }^{2}$ Young et al. (1989), ${ }^{3}$ Soifer et al. (1989), ${ }^{4}$ Moshir et al. (1990). The mm fluxes from ${ }^{5}$ Dumke et al. (1997), ${ }^{6}$ Guelin et al. (1993).

\begin{tabular}{clrrrrrrrc}
\hline Galaxy & $\begin{array}{l}\text { Hubble } \\
\text { type }\end{array}$ & $\begin{array}{r}\text { distance } \\
(\mathrm{Mpc})\end{array}$ & $\begin{array}{r}\text { dust mass } \\
\left(M_{\odot} \times 10^{7}\right)\end{array}$ & $\begin{array}{c}F_{60} \\
(\mathrm{Jy})\end{array}$ & $\begin{array}{c}F_{100} \\
(\mathrm{Jy})\end{array}$ & $\begin{array}{c}F_{450} \\
(\mathrm{Jy})\end{array}$ & $\begin{array}{c}F_{850} \\
(\mathrm{Jy})\end{array}$ & $\begin{array}{c}F_{1200} \\
(\mathrm{Jy})\end{array}$ & $\begin{array}{c}F_{1300} \\
(\mathrm{Jy})\end{array}$ \\
\hline NGC 891 & $\mathrm{Sb}$ & 9.5 & 5.6 & $50.5^{1}$ & $126^{1}$ & $32^{1}$ & $4.62^{1}$ & - & $0.85^{6}$ \\
NGC 5907 & $\mathrm{Sc}$ & 11.0 & 1.5 & $16.3^{2}$ & $55.9^{2}$ & - & - & $0.54^{5}$ & - \\
NGC 4013 & $\mathrm{Sbc}$ & 11.6 & 0.45 & $7.0^{3}$ & $23.1^{3}$ & - & - & - & - \\
UGC 1082 & $\mathrm{Sb}$ & 37.0 & 0.99 & $1.6^{4}$ & $3.7^{4}$ & - & - & - & - \\
UGC 2048 & $\mathrm{Sb}$ & 31.5 & 3.5 & $1.7^{4}$ & $3.5^{4}$ & - & - & - & - \\
\hline
\end{tabular}

${ }^{1}$ The flux densities from this reference are integrated in the range $\pm 225^{\prime \prime}$ along the major axis of the galaxy.

factor $F$ (in the absence of high resolution images) by the FIR colour. In the analysis of the five galaxies in subsequent sections, we refer to the following variants of the model:

1. The simple model: The simplest possible model takes into account only the information from the optical observations, i.e. the dust and old stellar distribution in the old disk without massive UV-emitting stars. The sole purpose of the "simple model" is to demonstrate that the old stellar population can not adequately heat the dust to account for the observed FIR luminosity;

2. The standard model: This is the "simple model" supplemented by a disk population of UV-emitting stars without associated dust, but with localised FIR emission. The free parameters are the SFR and the factor $F$;

3. The two-dust-disk model: This is the "standard model" supplemented by the presence of dust in the young stellar disk. The free parameters are the SFR, the factor $F$, and the dust mass in the "young dust disk".

The predictions of the model calculations are compared with available FIR, submm and $\mathrm{mm}$ data from the literature. In Table 1 we summarise the data used for comparison in the present work. We adopted $20 \%$ errors in the IRAS and IRAM data. For the SCUBA data we adopted the errors quoted by Alton et al. (1998). In Table 1 we also give some basic information on the properties of the sample galaxies. One factor in the choice of these galaxies for the radiation transfer analysis by X97 and X99 was their prominent dust lanes.

\section{Results for the "standard model"}

To demonstrate the need for a population of massive stars to account for the observed FIR luminosities in all five galaxies, we first calculated SEDs for the "simple model", in which the stellar and dust distributions are completely determined from the optical-NIR data as described in Sect. 2. These are plotted in the left column of Fig. 1 In all cases, the predicted FIR flux densities in the 60 and $100 \mu \mathrm{m}$ IRAS bands fall short of the observed luminosities by factors of between 5 and 10, showing that the heating of the dust from the old stellar population cannot account for the FIR emission. The results of the "simple model" are summarised in the second column of Table $2\left(L_{\text {simple }}\right)$, where we give the FIR luminosity of the galaxies that can be attributed to the old stellar population.

Assuming that the optically determined dust content we used for each galaxy is exact, the "standard model" can account for the observed FIR luminosity by transforming non-ionizing UV radiation from massive stars into the FIR through a combination of local absorption and absorption in the diffuse old disk, controlled by the factor $F$. If all the young stellar luminosity is absorbed locally $(F=1)$, then the efficiency of the transformation from non-ionizing UV to the FIR is unity, as for starburst galaxies. In the total absence of local absorption $(F=0)$ the global absorption efficiency is lower, with a value depending on the relative geometries of the diffuse dust and the massive stars. In principle, the factor $F$ can be determined from the FIR colours.

We investigated the combinations of $F$ and SFR for which the "standard model" was consistent with the IRAS 60 and $100 \mu \mathrm{m}$ measurements. For NGC 891 and UGC 2048, good agreement between model and data could be found for values of $F$ well above zero. However, for the remaining galaxies, the IRAS colours required $F$ values of zero or close to zero. This would imply high current star-formation rates and an amplitude in the UV stellar emissivity which would not join up smoothly with the empirically determined $B$ band stellar emissivities. Consequently, we have plotted in Fig. 1 the model prediction corresponding to the maximum value of $F$ still consistent with the IRAS colours. This also gives a monotonically increasing SED for the UV-optical stellar emissivities. Corresponding FIR bolometric luminosities ( $\left.L_{\text {standard }}\right)$, SFRs and factors $F$ are given in Table 2, Cols. 3-5 respectively. We emphasise however, that in principle one could have a non-steady SFR, so that the tabulated SFR are at the same time lower limits in SFR and upper limits for the factor $F$. Therefore we can discuss the results for the "standard model" also in terms of a range of possible values for $F$ and SFR, which will be compatible with the range in the IRAS errors.

We remark that a comparison of the values in the second and third column of Table 2 reveals that, according to 
Table 2. Results for the "standard model". $L_{\text {simple denotes }}$ the bolometric re-radiated luminosity when only the old stellar population $(B, V, I, J, K$ bands) is taken into account (i.e. for the "simple model"). $L_{\text {standard }}$ denotes the bolometric reradiated luminosity obtained by integrating the solution for the "standard model" as plotted in Fig. 1. Columns 4 and 5 contain the corresponding SFRs and factors $F$.

\begin{tabular}{ccccc}
\hline Galaxy & $\begin{array}{c}L_{\text {simple }} \\
W \times 10^{35}\end{array}$ & $\begin{array}{c}L_{\text {standard }} \\
W \times 10^{35}\end{array}$ & $\begin{array}{c}\text { SFR } \\
M_{\odot} \mathrm{yr}^{-1}\end{array}$ & $F$ \\
\hline NGC 891 & 10.8 & 48.1 & 3.48 & 0.28 \\
NGC 5907 & 3.7 & 15.5 & 2.17 & 0.08 \\
NGC 4013 & 2.0 & 11.0 & 1.23 & 0.19 \\
UGC 1082 & 2.3 & 21.3 & 2.59 & 0.23 \\
UGC 2048 & 5.7 & 18.3 & 1.36 & 0.27 \\
\hline
\end{tabular}

the standard model, only $10 \%$ to $30 \%$ of the dust emission is powered by the old stellar population.

In the above we have made the assumption that the "standard model" is a sufficiently realistic model of the galaxies. Some evidence that this may not be the case is apparent from Fig. 1 (right column), where all the model spectra peak at around $120 \mu \mathrm{m}$, well shortward of the typical peak near $150 \mu \mathrm{m}$ seen in spiral galaxies. This may be an indication that the overall optical depth of the galaxies is underestimated by the "standard model", indicating the presence of a further dust component in the plane of each galaxy. This component may either be smoothly distributed or be in the form of optically thick clumps or some combination of these. For the two galaxies that submm and/or mm measurements are available (NGC 891 and NGC 5907), this is explicitly confirmed by the undershoot of the "standard model" prediction in the submm and $\mathrm{mm}$ regime. In Paper I, we used the available submm fluxes to quantify the missing dust and introduced the "two-dust-disk model", which successfully reproduced the spectrum and the spatial distribution of the FIR emission in NGC 891. In the next section we will apply the "twodust-disk model" to NGC 5907 to determine the total dust content of this galaxy and examine the effect of this more realistic case on the derived SFRs.

\section{Application of the "two-dust-disk model" to NGC 5907}

In Paper I we proved that in NGC 891 the amount of dust (on the basis of the grain optical constants adopted) quoted by X99 is underestimated by a factor of two, and that the addition of a second dust disk, that follows the spatial distribution of the young stellar population, reproduces very well the observed FIR luminosity and the radial profiles observed by Alton et al. (1998). For NGC 5907, the measurement at $1200 \mu \mathrm{m}$ by Dumke et al. (1997) can be used to constrain the quantity of dust in the second disk.

Inspection of Fig. 1 (right column) shows that the "standard model" solution for $F=0.08$, which is in agreement with the IRAS points, crassly undershoots the
$1200 \mu \mathrm{m}$ point. As for NGC 891, the only way to account for both the IRAS and the $1200 \mu \mathrm{m}$ observations is to include more dust in our model, as simply increasing the star-formation rate would provide too much luminosity in the IRAS range.

The second-dust-disk's spatial distribution was constrained to follow that of the young stellar population, which is taken to be exponential with scalelength and scaleheight $h_{\mathrm{d}}=5020 \mathrm{pc}$ and $z_{\mathrm{d}}=90 \mathrm{pc}$ respectively (see Sect. 2). To find the solution, the total amount of dust in the second dust disk was varied jointly with the SFR until the FIR-submm SED of our model fitted all three measurements. In Fig. 2 we present the resulting spectrum overlaid with the data. This spectrum corresponds to a $\mathrm{SFR}=2.2 M_{\odot} \mathrm{yr}^{-1}$ and $F=0.10$. The total dust mass in the second dust disk is $4.5 \times 10^{7} M_{\odot}$. By comparison, the dust mass quoted for the old disk of NGC 5907 by X99 is $1.5 \times 10^{7} M_{\odot}$.

Thus, we have had to add 3 times more dust over the quantity implied by the observed dust lane to obtain agreement with the data on the basis of the "two-dust-disk model". The derived value of $F$ is still very low, but the spectrum of the diffuse component is too warm (NGC 5907 is more optically thin than NGC 891) to allow the addition of a warm component related to HII regions. While we could have produced a colder spectrum for the diffuse emission, allowing some room for HII regions, this would have been at the cost of adding yet more dust in the second disk. A larger quantity of dust in the second disk does not seem very likely, as the total dust mass for the fitted $F=0.10$ case already implies a gas-to-dust ratio of 130 by mass (instead of 520 according to X99). As a comparison, the gas-to-dust ratio in the Milky Way is 133 .

The total IR-submm re-radiated luminosity of NGC 5907, obtained by integrating the $F=0.10$ "twodust-disk model" SED, is $50.5 \times 10^{35} \mathrm{~W}$ out of which $27.0 \times 10^{35} \mathrm{~W}$ is attributed to heating from the young stellar population. Thus, about $40 \%$ of the dust emission is powered by the old stellar population. More dust in the "young dust disk", a lower SFR and a higher factor $F$ would increase the disk opacity and thus the contribution of the old stellar population, but we excluded this solution due to the high dust-to-gas ratio. The $40 \%$ contribution from the old stellar population represents an upper limit, since a possible clumpiness in the dust distribution would decrease the absorption efficiency in the optical band.

On the basis of the model's inherent assumption, that all the dust (in both disks) of the galaxy is distributed smoothly, the central face-on optical depth in the optical band is $\tau_{\mathrm{v}}=1.4$ for NGC 5907 .

The major difference between NGC 891 and NGC 5907, on the basis of the "two-dust-disk model", is that the spectrum of the former apparently allows for the existence of a larger contribution from HII regions. Presumably NGC 5907 must have HII regions, and there is no obvious reason why the local properties of these HII regions, in particular the probability of local absorption of the UV radiation, should differ between the two systems. 

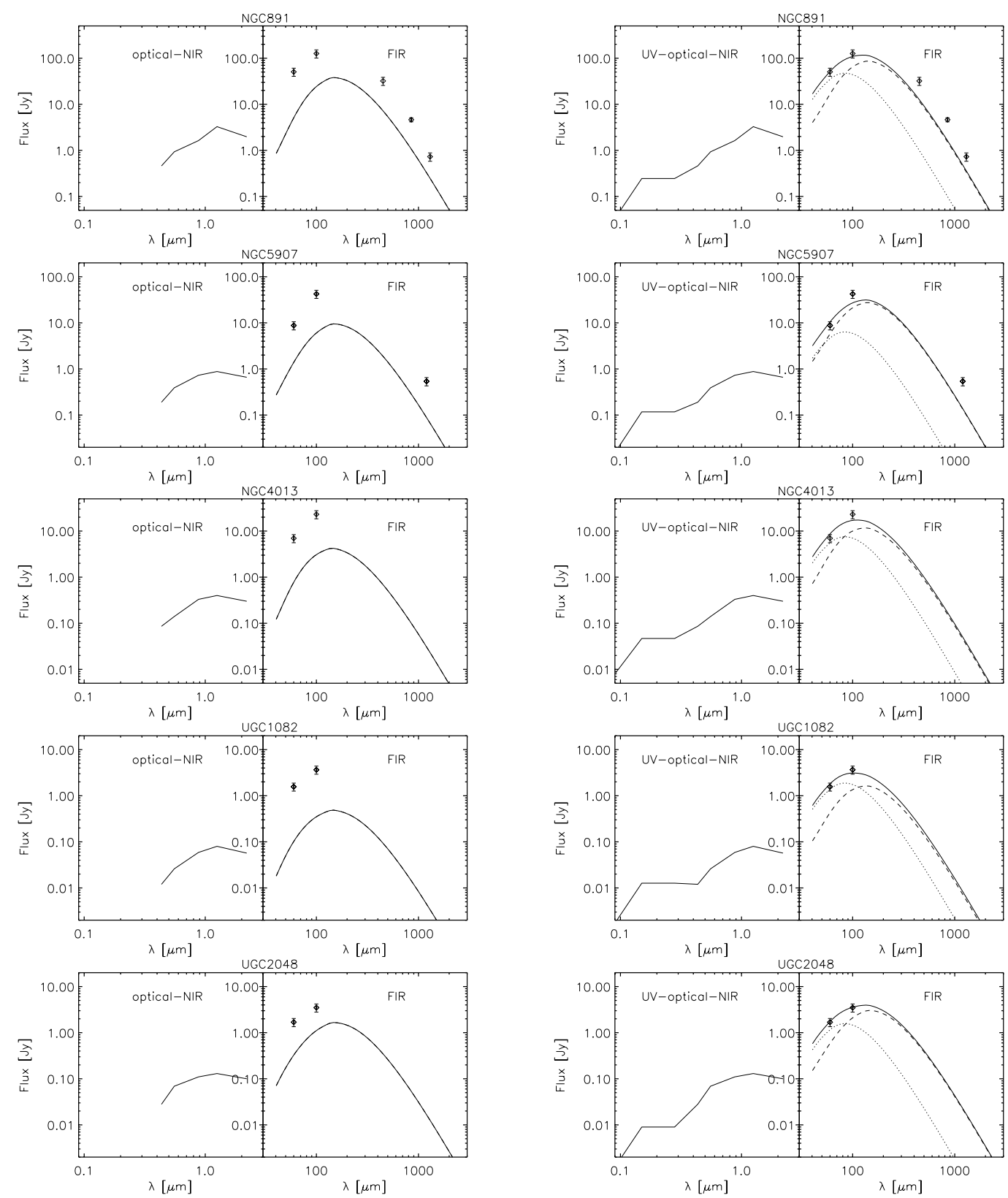

Fig. 1. The SED of the 5 galaxies. In the left column the SED is derived for the "simple model". In the right column the SED is derived for the "standard model". The predicted FIR emission from the diffuse disk is plotted with dashed lines and the contribution of localised sources with dotted lines. The corresponding SFR and factor $F$ for each galaxy are given in Table 2 . For NGC 891 and UGC 2048, the model corresponding to the value of $F$ which best fits the IRAS colour is plotted. In the cases of NGC 5907, NGC 4013 and UGC 1082, the model corresponding to the maximum value of $F$ still consistent with the IRAS colour is plotted (see text).

One potential way out of this difficulty might be to suppose that the spectral template for the HII regions used in our model underestimates the associated submm emission. As stated in Paper I, we did not attempt to include potential cold dust emission components that might be expected from "parent" molecular clouds in juxtaposition to their "offspring" HII regions. These might contribute a significant fraction of the observed submm emission, but be relatively faint in the IRAS range. If so, the dust content and the luminosity of the diffuse disk would be lower for both galaxies, but more so for the more optically thin diffuse disk of NGC 5907. This would make room for larger values of $F$ in the model fits, particularly for NGC 5907.

An alternative scenario, also discussed in Paper I, would be the presence of truly quiescent cold dust clouds in the disk not associated with HII regions. In that case, the difference between the two galaxies might indicate a greater star-formation activity in the disk of NGC 891, in the sense that a larger proportion of the cold dust clouds in the disk had been triggered into massive star formation. 


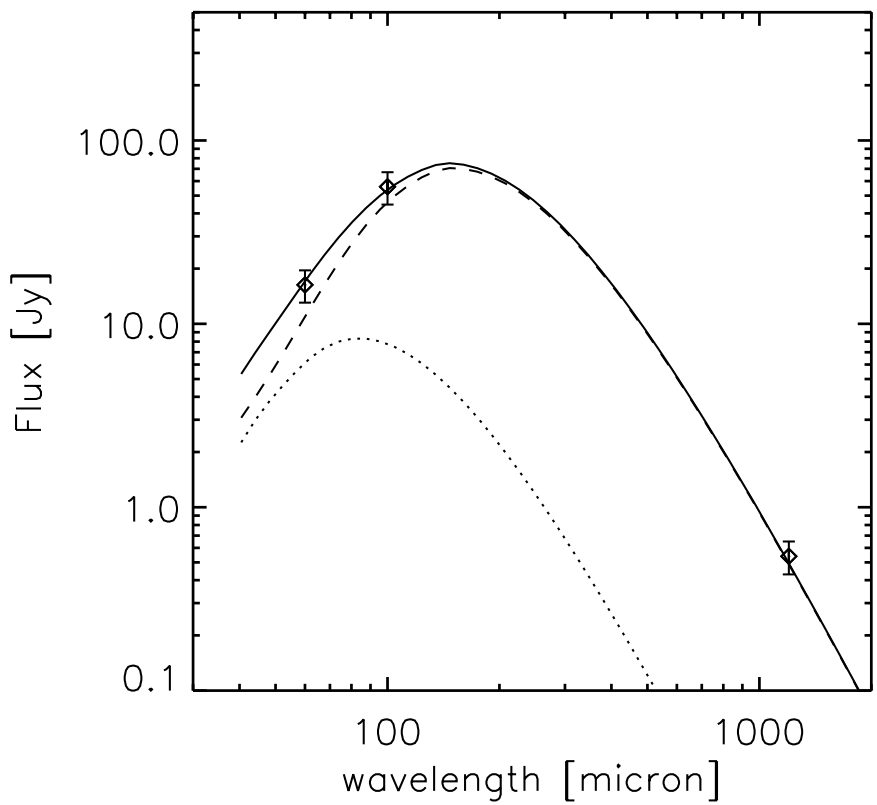

Fig. 2. SED of the "two-dust-disk model" for NGC 5907 for $F=0.10$ and $\mathrm{SFR}=2.2 M_{\odot} \mathrm{yr}^{-1}$, overlaid with data points given in Table 1 . The predicted FIR emission from the diffuse disk is plotted with a dashed line and the contribution of localized sources with a dotted line.

\section{Discussion}

Our results confirm that it is a common tendency for edge-on galaxies to hide a significant fraction of their dust from optical extinction studies. In two cases where subor near-mm data are available (NGC 891, as analyzed in Paper I and NGC 5907 in this work), we have modeled the FIR-submm SED assuming that the additional dust is distributed in a second diffuse disk associated with the young stellar population. We have found that the SEDs could be fitted in terms of total dust masses of respectively twice and four times the masses inferred by X99 from the optical radiation transfer analysis for NGC 891 and NGC 5907. The corresponding SFRs and local non-ionizing UV absorption factors $F$ in the best fits were $\mathrm{SFR}=3.8 M_{\odot} \mathrm{yr}^{-1}$ and $F=0.22$ for NGC 891 and $\mathrm{SFR}=2.2 M_{\odot} \mathrm{yr}^{-1}$ and $F=0.10$ for NGC 5907 .

The main uncertainty in the inferred SFRs is the value of the factor $F$. In principle, $F$ can be determined from the colour of the FIR SED, especially the $60 / 100 \mu \mathrm{m}$ colour ratio. However, as discussed in Sect. 4, the value of the factor $F$, which can be fitted, in practice is constrained by the assumption of the "two-dust-disk model" that all the additional dust needed to fit the submm measurements is diffuse. In reality, the geometry of the additional dust could be, as emphasised in Paper I, in the form of optically thick clumps associated with the HII regions. In this case the inferred SFRs would, depending on the factor $F$, differ from those determined on the assumption of a purely diffuse dust distribution without local absorption. However, at present, the available data does not allow us to quantify the clumpy component of UV-absorbing dust.
The SFRs and factors $F$ inferred from the "standard model" for NGC 891 and NGC 5907 in Sect. 3 (where the dust content and distribution is determined from X99's optical analysis) hardly differs from the values determined from the "two-dust-disk model" applied to NGC 891 and NGC 5907 in Sect. 4. This is at first sight surprising, as one would expect lower SFRs due to the higher opacity of the diffuse disk in the "two-dust-disk model". However, the SFR determined from the "standard model" was obtained by fitting the SEDs exclusively to the IRAS 60 and $100 \mu \mathrm{m}$ measurements common to the sample of the 5 galaxies. The reason that the SFRs determined from the "standard model" are so similar to those derived from the "two-dust-disk model" is simply that the additional submm luminosity arises from the extra dust (from the second dust disk) rather than from additional SFR. As remarked in Sect. 3, the rather warm SEDs for all the "standard model" fits suggest that additional dust is present in the disks of all 5 galaxies.

To statistically evaluate our results for SFR, we make the assumption that the SFRs determined from the "standard model" fits to the IRAS 60 and $100 \mu \mathrm{m}$ measurements for our sample of 5 galaxies give, as is the case of NGC 891 and NCG 5907, a reasonable estimate of the SFR which would have been obtained with an analysis based on the "two-dust-disk model". We can then compare the SFR characteristics of the 5 edge-on galaxies with the larger sample of 61 galaxies with inclinations less than 75 degrees which were studied by Kennicutt (1998) on the basis of $\mathrm{H}_{\alpha}$ measurements.

Figure 3 depicts the relation between the disk-averaged surface density in SFR $\left(\Sigma_{\mathrm{SFR}}\right)$ determined from the "standard model" as a function of the average gas surface density $\left(\Sigma_{\mathrm{g}}\right)$ for the 5 galaxies. The plotted data is summarized in Table 3 . The $\Sigma_{\mathrm{SFR}}$ is calculated from the SFRs determined from the "standard model" and listed in Table 2. The full range in conceivable SFRs is given by the vertical error bars. The upper and lower limits for the SFRs are calculated such that the predicted SED is still consistent with the IRAS colours (within the 20\% IRAS error bars). Lower error bars are not given when the plotted SFRs represent lower limits for the SFR (maximum values for the factors $F$, see discussion in Sect. 3). In these cases lower limits would be possible only if we allowed for different sources of uncertainties, like variations in the spectral shape of the template used for the HII regions. However this is hard to quantify, and in the following we assume that the errors of the SFR are given only by the uncertainties in the IRAS data.

The $\Sigma_{\mathrm{g}}$ were calculated from the gas masses taken from García-Burillo et al. (1992) and Rupen (1991) respectively for $\mathrm{H}_{2}$ and $\mathrm{HI}$ in NGC 891, from Dumke et al. (1997) for $\mathrm{H}_{2}$ and $\mathrm{HI}$ in NGC 5907, from Bottema (1995) and Gomez \& Garcia (1997) for $\mathrm{H}_{2}$ and $\mathrm{HI}$ in NGC 4013, from Giovanelli \& Haynes (1993) for HI in UGC 1082 and from Huchtmeier \& Richter (1989) for HI UGC 2048. In the last two cases, where only data for HI was available, we estimated the total gas mass by doubling the HI gas mass. 


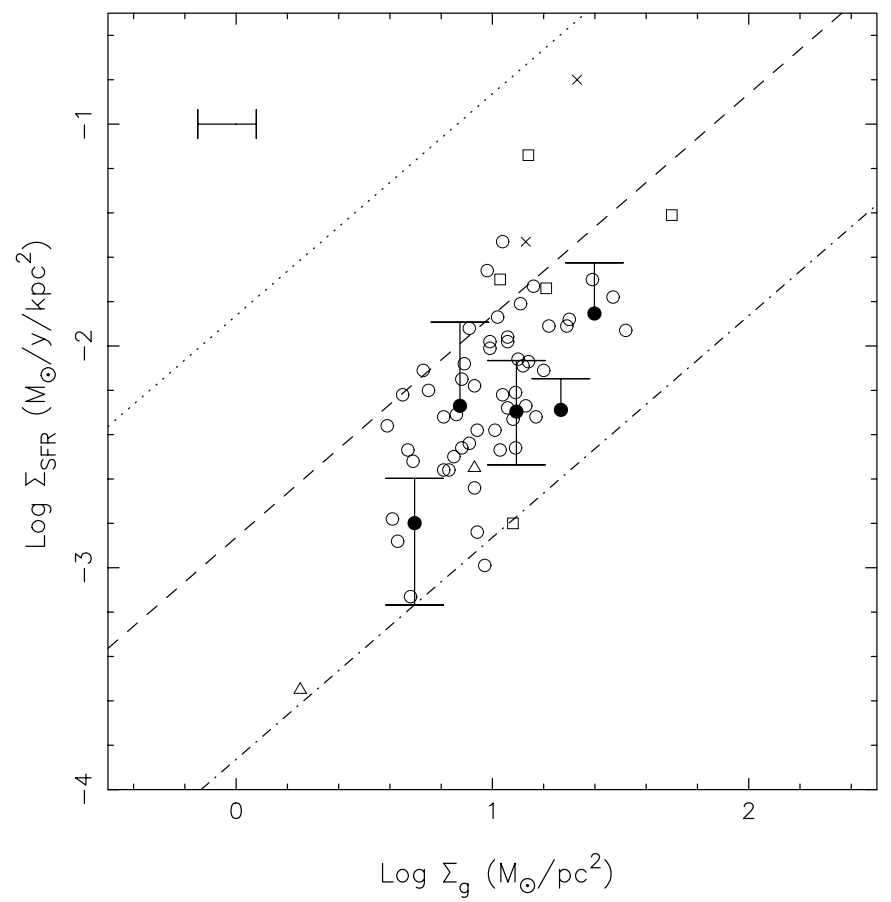

Fig. 3. Disk-averaged SFR surface density $\left(\Sigma_{\mathrm{SFR}}\right)$ as a function of average gas surface density $\left(\Sigma_{\mathrm{g}}\right)$ for our galaxy sample and for the sample of Kennicutt (1998) of 61 normal disk galaxies with $\mathrm{SFR}$ determined from $\mathrm{H}_{\alpha}$ measurements. The 5 galaxies from our sample are plotted as filled circles and the corresponding $\Sigma_{\mathrm{SFR}}, \Sigma_{\mathrm{SFR}}^{\min }, \Sigma_{\mathrm{SFR}}^{\max }, \Sigma_{\mathrm{g}}$ and disk areas are listed in Table 3. The SFR surface densities were calculated by averaging the SFRs determined from the "standard model" (Sect. 3) over disks with an optically defined boundary $\left(R_{\mathrm{o}}\right)$ taken to be 3 times the intrinsic radial scalelength $h_{\mathrm{s}}$ determined from the radiation transfer modeling in the $I$ band. The galaxies from the sample of Kennicutt are plotted as open circles ( $\mathrm{Sb}, \mathrm{Sc}, \mathrm{SBb}$, $\mathrm{SBc}$ ), triangles (Sa), open squares (Unknown/Not Available), crosses (Irr). The dotted, dashed and dot-dashed lines represent star-formation efficiencies corresponding to consumptions of 100,10 and 1 percent of the gas mass in $10^{8} \mathrm{yr}$.

The horizontal error bar corresponds to the uncertainty in the gas masses for which we adopted an average 0.2 dex error. The surface area of the disk was calculated for $R_{\mathrm{O}}=$ $(3 \pm 0.5) h_{\mathrm{d}}$, where $h_{\mathrm{d}}$ is the intrinsic radial scalelength determined from the radiation-transfer modeling in the $I$ band. In their analysis of surface photometry of the outer regions of spiral disks, Pohlen et al. (2000) show that the disk boundaries are typically in this range.

The points for the 5 galaxies in Fig. 3 lie within the area of the diagram occupied by the galaxies in the Kennicutt sample. The match is even better for those members of the Kennicutt sample with Hubble types Sb to Sc. This agreement is quite reassuring, bearing in mind the several factors which could introduce a systematic difference between the SFRs inferred for a sample of nearly face-on systems from $\mathrm{H}_{\alpha}$ measurements compared with the present technique for edge-on systems based on an analysis of broad-band non-ionizing UV re-radiated in the FIR-submm range.
Firstly, the $\mathrm{H}_{\alpha}$ analysis is sensitive to the most massive stars and in particular to the assumed mass cut of $100 M_{\odot}$. Whereas the FIR-submm modeling also assumes the same mass cut in the conversion of SFR to non-ionizing luminosity (see Sect. 2 and Paper I), our model is less sensitive to this effect.

Secondly, whereas the $\mathrm{H}_{\alpha}$ is sensitive to the starformation history of the last $10^{7} \mathrm{yr}$, our broad-band FIR-submm SED analysis samples approximately the last $10^{8} \mathrm{yr}$. Thus, our analysis is consistent with the basic hypothesis (see Kennicutt 1998) for "normal" spiral galaxies of a steady star-formation activity. In principle, we could extend our analysis based on our determinations of the intrinsic populations and use the determined intrinsic colours to determine more accurately the SFR history of the galaxies, though this is beyond the scope of this paper.

The assumption of a steady-state star-formation rate is also broadly consistent with the timescales for the exhaustion of the current gas supply under the derived SFRs. The dotted, dashed and dot-dashed lines in Fig. 3 represent star-formation efficiencies corresponding to consumptions of 100,10 and 1 percent of the gas mass in $10^{8} \mathrm{yr}$. We note that in constructing Fig. 3, we have assumed that the HI line emission, from which the gas masses for the 5 edge-on galaxies were in part determined, have not been affected by self absorption in the $21 \mathrm{~cm}$ line. The effect of self-absorption would be to shift the points for the 5 edgeon galaxies to the left of the positions corresponding to their actual gas surface densities in Fig. 3.

Thirdly, the SFRs derived from $\mathrm{H}_{\alpha}$ were corrected by a single factor for extinction, despite the varying orientations. As well as possibly affecting the vertical position of the galaxies on the plot, this may induce some scatter, especially if all the dust were diffusely distributed. The systematic effect may be expressed in terms of the factor $F$ : an overestimation of the factor $F$ is equivalent to an overestimation of the local extinction in the star-formation regions (statistically averaged over the population of HII regions in a disk). Thus, while moving to higher factors $F$ would move the points for the 5 galaxies towards lower SFRs, it would have the opposite effect for the SFRs determined from the $\mathrm{H}_{\alpha}$.

Lastly, we remark that NGC 891 does not appear as an exceptional system compared with the other 4 galaxies in our sample (and with Kennicutt's 1998, normal galaxy sample) on the basis of SFR normalized to disk area. Our work thus provides no evidence that this galaxy's exceptional layer of extraplanar $\mathrm{H}_{\alpha}$-emitting diffuse ionizing gas (e.g. Hoopes et al. 1999) and surrounding X-ray-emitting hot gas (e.g. Bregman \& Houck 1997) is attributable to unusual star-formation activity.

\section{Summary}

- This paper is the second part on a series of papers dedicated to the modeling of the SEDs of disk galaxies. In Paper I we described a new tool for the analysis of the UV to the submm SED and applied this tool 
Table 3. The average gas surface density $\left(\Sigma_{\mathrm{g}}\right)$ and diskaveraged SFR surface density $\left(\Sigma_{\mathrm{SFR}}\right)$ for our galaxy sample. The lower and upper limits in the $\Sigma_{\mathrm{SFR}}$ are also given together with the disk area used to normalize the SFRs and gas masses.

\begin{tabular}{rcrrrr}
\hline Galaxy & $\log \Sigma_{\mathrm{g}}$ & $\log \Sigma_{\mathrm{SFR}}$ & $\log \sum_{\mathrm{SFR}}^{\min }$ & $\log \sum_{\mathrm{SFR}}^{\max }$ & Area \\
& $\frac{M_{\odot}}{\mathrm{pc}^{2}}$ & $\frac{M_{\odot}}{\mathrm{yr} \mathrm{kpc}^{2}}$ & $\frac{M_{\odot}}{\mathrm{yr} \mathrm{kpc}^{2}}$ & $\frac{M_{\odot}}{\mathrm{yr} \mathrm{kpc}^{2}}$ & $\mathrm{kpc}^{2}$ \\
\hline NGC 891 & 1.09 & -2.30 & -2.54 & -2.07 & 687 \\
NGC 5907 & 1.27 & -2.29 & -2.29 & -2.15 & 421 \\
NGC 4013 & 1.40 & -1.85 & -1.85 & -1.63 & 88 \\
UGC 1082 & 0.87 & -2.27 & -2.27 & -1.89 & 482 \\
UGC 2048 & 0.70 & -2.80 & -3.17 & -2.60 & 855 \\
\hline
\end{tabular}

to the well known nearby edge-on galaxy NGC 891. In the present paper we have extended the analysis from Paper I to a sample of four additional edge-on galaxies (NGC 5907, NGC 4013, UGC 1082, UGC 2048) and proved that the solution obtained for NGC 891 is generally valid. We have also shown that NGC 891 is not an exceptional system in terms of its SFR density;

- In all the systems the dust is predominantly heated by the young stellar population. The contribution of the old stellar population can account for at most $40 \%$;

- The SFRs derived from our "standard model" are consistent with the SFRs derived from the more sophisticated "two-dust-disk model" and are also consistent with the Kennicutt's (1998) Schmidt law for disk galaxies.

Acknowledgements. One of us (A.M.) thanks the Max Planck Institut für Kernphysik for financial support and hospitality during his visit to Heidelberg.

\section{References}

Alton, P. B., Bianchi, S., Rand, R. J., et al. 1998, ApJ, 507, L125

Bianchi, S., Davies, J. I., Alton, P. B., Gerin, M., \& Casoli, F. 2000, A\&A, 353, L13

Bianchi, S., Davies, J. I., \& Alton, P. B. 2000, A\&A, 359, 65

Bottema, R. 1995, A\&A, 295, 605

Bregman, J. N., \& Houck, J. C. 1997, ApJ, 485, 159

Bruzual, A. G., \& Charlot, S. 2001, in preparation

Devriendt, J. E. G., Guiderdoni, B., \& Sadat, R. 1999, A\&A, 350,381

Dumke, M., Braine, J., Krause, M., et al. 1997, A\&A, 325, 124
Dunne, L., Eales, S., Edmunds, M., et al. 2000, MNRAS, 315, 115

Efstathiou, A., Rowan-Robinson, M., \& Siebenmorgen, R. 2000, MNRAS, 313, 375

García-Burillo, S., Guélin, M., Cernicharo, J., \& Dahlem, M. 1992, A\&A, 266, 21

Giovanelli, R., \& Haynes, M. P. 1993, AJ, 105, 1271

Gomez de Castro, A. I., \& García-Burillo, S. 1997, A\&A, 322, 381

Guelin, M., Zylka, R., Mezger, P. G., et al. 1993, A\&A, 279, L37

Haas, M., Lemke, D., Stickel, M., et al. 1998, A\&A, 338, L33

Hoopes, C. G., Walterbos, R. A. M., \& Rand, R. J. 1999, ApJ, 522,669

Huchtmeier, W. K., \& Richter, O. G. 1989, A general catalog of HI observations of galaxies (New York, Springer-Verlag)

Israel, F. P., ven der Werf, P. P., \& Tilanus, R. P. J. 1999, A\&A, 344, L83

Kennicutt, R. C. Jr. 1998, ApJ, 498, 541

Kuchinski, L. E., Terndrup, D. M., Gordon, K. D., \& Witt, A. N. 1998, AJ, 115, 1438

Kylafis, N. D., \& Bahcall, J. N. 1987, ApJ, 317, 637

Laor, A., \& Draine, B. T. 1993, ApJ, 402, 441

Mathis, J. S., Rumple, W., \& Nordsieck, K. H. 1977, ApJ, 217, 425

Misiriotis, A., Kylafis, N. D., Papamastorakis, J., \& Xilouris, E. M. 2000, A\&A, 353, 117

Moshir, M., et al. 1990, IRAS Faint Source Catalogue.

Neininger, N., Guélin, M., García-Burillo, S., et al. 1996, A\&A, 310,725

Ohta, K., \& Kodaira, K. 1995, PASJ, 47, 17

Pohlen, M., Dettmar, R.-J., \& Lutticke, R. 2000, A\&A, 357, 1

Popescu, C. C., Misiriotis, A., Kylafis, N. D., Tuffs, R. J., \& Fischera, J. 2000, A\&A, 362, 138, Paper I

Rupen, M. P. 1991, AJ, 102, 48

Silva, L., Granato, G. L., Bressan, A., \& Danese, L. 1998, ApJ, 509, 103

Soifer, B. T., Boehmer, L., Neugebauer, G., \& Sanders, B. 1989, AJ, 98, 766

Stickel, M., Lemke, D., Klaas, U., et al. 2000, A\&A, 359, 865

Xilouris, E. M., Kylafis, N. D., Papamastorakis, J., Paleologou, E. V., \& Haerendel, G. 1997, A\&A, 325, 135 (X97)

Xilouris, E. M., Alton, P. B., Davies, J. I., et al. 1998, A\&A, 331, 894 (X98)

Xilouris, E. M., Byun, Y. I., Kylafis, N. D., Paleologou, E. V., \& Papamastorakis, J. 1999, A\&A, 344, 868 (X99)

Xu, C., \& Buat, V. 1995, A\&A, 293, L65

Xu, C., \& Helou, G. 1996, ApJ, 456, 163

Young, J. S., Xie, S., Kenney, J. D. P., \& Rice, W. L. 1989, ApJS, 70, 699 